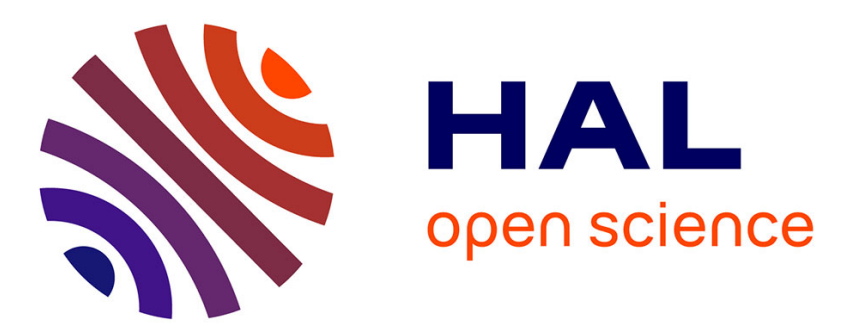

\title{
Virtual Retina : a biological retina model and simulator, with contrast gain control
}

Adrien Wohrer, Pierre Kornprobst, Thierry Viéville

\section{To cite this version:}

Adrien Wohrer, Pierre Kornprobst, Thierry Viéville. Virtual Retina : a biological retina model and simulator, with contrast gain control. [Research Report] RR-6243, INRIA. 2007, pp.32. inria00160716v2

\section{HAL Id: inria-00160716 https://hal.inria.fr/inria-00160716v2}

Submitted on 10 Jul 2007

HAL is a multi-disciplinary open access archive for the deposit and dissemination of scientific research documents, whether they are published or not. The documents may come from teaching and research institutions in France or abroad, or from public or private research centers.
L'archive ouverte pluridisciplinaire HAL, est destinée au dépôt et à la diffusion de documents scientifiques de niveau recherche, publiés ou non, émanant des établissements d'enseignement et de recherche français ou étrangers, des laboratoires publics ou privés. 
INSTITUT NATIONAL DE RECHERCHE EN INFORMATIQUE ET EN AUTOMATIQUE

\section{Virtual Retina : a biological retina model and simulator, with contrast gain control}

Adrien Wohrer — Pierre Kornprobst — Thierry Viéville

$N^{\circ} 6243$

Juillet 2007

Thème BIO

apport

de recherche 



\title{
Virtual Retina : a biological retina model and simulator, with contrast gain control
}

\author{
Adrien Wohrer* ${ }^{*}$ Pierre Kornprobst ${ }^{\dagger}$, Thierry Viéville \\ Thème BIO — Systèmes biologiques \\ Projet Odyssée
}

Rapport de recherche $n^{\circ} 6243$ - Juillet 2007 -32 pages

\begin{abstract}
A detailed retina model is proposed, that transforms a video sequence into a set of spike trains, as those emitted by retinal ganglion cells. It includes a linear model of filtering in the Outer Plexiform Layer (OPL), a contrast gain control mechanism modeling the non-linear feedback of some amacrine cells on bipolar cells, and a spike generation process modeling ganglion cells. A strength of the model is that each of its features can be associated to a precise physiological signification and location. The resulting retina model can simulate physiological recordings on mammalian retinas, including such non-linearities as cat Y cells, or contrast gain control. Furthermore, the model has been implemented on a large-scale simulator that can emulate the spikes of up to 100,000 neurons.
\end{abstract}

Key-words: Retina simulator, Contrast gain control, Conductances, Spikes

\footnotetext{
* Adrien.Wohrer@sophia.inria.fr

† Pierre.Kornprobst@ sophia.inria.fr

‡ Thierry.Vieville@sophia.inria.fr
} 


\section{Virtual Retina : modèle et simulateur de rétine, avec contrôle de gain}

Résumé : Ce rapport propose un modèle de rétine, qui prend en entrée une séquence video et la convertit en un ensemble de trains de potentiels d'action (ou spikes), à la manière des cellules ganglionnaires en sortie d'une rétine de mammifère. Le modèle possède trois composantes successives: un filtre linéaire qui modélise la couche plexiforme externe (OPL) de la rétine, un mécanisme de contrôle de gain non-linéaire modélisant une intéraction entre cellules bipolaires et amacrines, et un processus d'émission de potentiels d'action au-niveau des cellules ganglionnaires. Cette architecture possède un lien fort avec l'anatomie des rétines réelles, tout en permettant de reproduire avec précision certaines caractéristiques non-linéaires des cellules ganglionnaires (cellules Y, mécanisme de contrôle de gain). Enfin, le modèle a été implémenté sous la forme d'un simulateur à grande échelle, qui permet de simuler jusqu'à 100,000 cellules ganglionnaires.

Mots-clés : Rétine, Simulateur, Contrôle de gain, Conductances, Spikes 


\section{Contents}

1 Introduction $\quad 4$

2 Methods 5

2.1 General structure of the model . . . . . . . . . . . . . . . . . 5

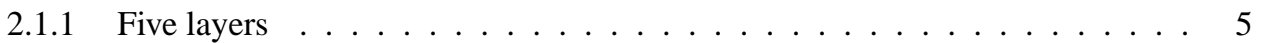

2.1.2 Horizontal couplings . . . . . . . . . . . . . . . . 6

2.2 Outer Plexiform Layer . . . . . . . . . . . . . . . . . . . . . . . . 6

2.2.1 Center-surround organization in the OPL . . . . . . . . . . . . 8

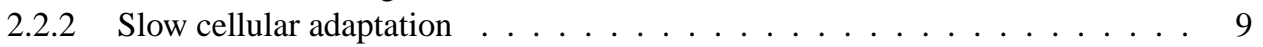

2.3 Inner Plexiform Layer . . . . . . . . . . . . . . . . . . . . . . . . . . . . . 9

2.3.1 Bipolar charging equation and the contrast gain control . . . . . . . . . . 10

2.3.2 Synaptic current upon ganglion cells . . . . . . . . . . . . . . . . 12

2.4 Ganglion layer . . . . . . . . . . . . . . . . . . . . . 13

2.4.1 Spike generation in ganglion cells . . . . . . . . . . . . . . . . 13

2.4.2 Ganglion cell sampling configurations . . . . . . . . . . . . . . . . 14

3 Results 15

3.1 Retina simulator customization . . . . . . . . . . . . . . . . 15

3.2 Multi-sinus experiment and the contrast gain control effect . . . . . . . . . . 16

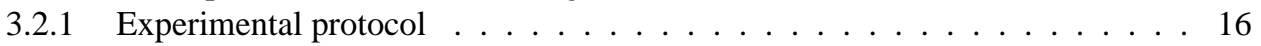

3.2.2 Experimental observation . . . . . . . . . . . . . . . . 17

3.2 .3 Simulation with our model . . . . . . . . . . . . . . . . . 18

3.3 Response of cat $\mathrm{X}$ and $\mathrm{Y}$ cells to grating apparitions . . . . . . . . . . . . . 18

3.3.1 Experimental protocol and observations . . . . . . . . . . . . . . . 19

3.3 .2 Simulation with our model . . . . . . . . . . . . . . . . 21

3.4 Results on real images . . . . . . . . . . . . . . . . . . . 21

3.4.1 Perceptual effect of contrast gain control . . . . . . . . . . . . . 21

3.4.2 Large-scale simulation of the model . . . . . . . . . . . . . . 22

4 Discussion 23

4.1 Non-linear gain controls . . . . . . . . . . . . . . . . . . . 23

4.1.1 Synaptic conductances . . . . . . . . . . . . . . . 23

4.1.2 Feedback mechanism? . . . . . . . . . . . . . . . 25

4.1.3 Other models of gain control . . . . . . . . . . . . . . . 26

4.2 Subclasses of ganglion cells . . . . . . . . . . . . . . . . . . . . . . . . . . . . . . . . . .

4.2.1 Ganglion cells in cat and primate retinas . . . . . . . . . . . . . . 27

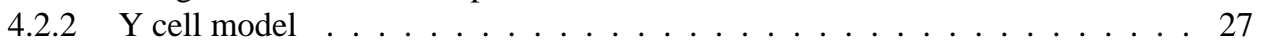

5 Conclusion $\quad 29$

$\mathrm{RR} n^{\circ} 6243$ 


\section{Introduction}

How does the retina transform incoming light into spike trains sent to the brain? To address this question, we propose a spiking retina simulator, called Virtual Retina, based on a detailed model of retinal physiology and reproducing some interesting behaviors of mammalian ganglion cells. This simulator is highly customizable and large-scale simulations (up to around 100,000 spiking cells) are possible (see Figure 1). This simulator will be an evolutionary tool for neuroscientists to analyze their measurements, but also to consider realistic spikes trains inputs in subsequent treatments.

Retinal processing is often modeled by three successive stages: Linear filtering on the visual stimulus, static non-linearity and then spike generation. This general architecture has been termed the retinal standard model by Carandini et al [4]. Virtual Retina is inspired by, and extends, such models. In particular, the first stage of our model is also a spatio-temporal linear filter, inspired by the Mahowald-Mead silicon retina [21] and subsequent linear models [14].

However, by opposition with standard models, Virtual Retina incorporates a non-linear mechanism of contrast gain control, directly inspired from retinal physiology. Furthermore, most standard models have an empirical architecture, designed to fit some experimental ganglion cells recordings $[5,17,12]$. Instead, we propose here a model where each part can be mapped to physiology, including the interpretation of most parameters.

Of course, we also validated our model by reproducing some experimental ganglion cell recordings $[10,28]$. The goal in doing so was twofold. First, naturally, to prove the simulation capabilities of our model. Second, to stress the (long-known) limitations of pure linear models, and explain how our model accounts for the different observed non-linearities. In this article we discuss three experimental non-linear effects in particular. First effect is contrast gain control, accounted for in our model by a feedback mechanism. Second effect is the spatial non-linearity of a particular type of ganglion cells, known as Y cells in the cat retina. Third effect is slow cellular adaptation, a characteristic shared to some extent by most real neurons.

Another biological fact that our model has to tackle is the distinction usually made in retinal physiology between the so-called tonic and phasic ganglion cells. The tonic-phasic opposition is a general concept in physiology that can be described in simple words: "A tonic process is one that continues for some time or indefinitely after being initiated, while a phasic process is one that shuts down quickly" [11]. In the retina, some ganglion cells have a long-lasting response after apparition of a static visual stimulus (tonic cells), while others only respond by a strong and short activation wave right after stimulus onset, and return to being silent afterward (phasic cells). Virtual Retina encompasses both types of cells, according to the value of a particular parameter.

The article is organized as follows. In Section 2, we detail the three stages of our retina model. The first stage is a linear filter that reproduces the center-surround architecture as arising from the interaction between light receptors and horizontal cells. The second stage is the main contribution of this paper in terms of retinal modeling: a contrast gain control mechanism at the level of bipolar cells, as driven by feedback leak conductances from a certain class of amacrine cells onto bipolar cells. The third stage of the model is the spike generation, occurring in the layer of ganglion cells. The model is tested in Section 3, through its implementation by the simulator Virtual Retina. First, we validate our model by comparing its spiking output with recordings of ganglion cells in different 


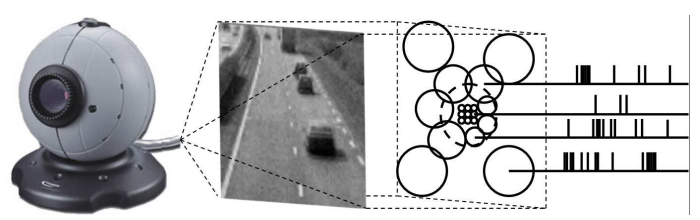

Figure 1: Overview of the system: The simulator Virtual Retina transforms a video stream into a set of spikes. The strength of the simulator is to reproduce as much as possible mammalian retina characteristics and dynamical properties such as contrast gain control.

experiments. Then, we present large-scale simulations. In Section 4, we compare our retinal model with existing models, including recent models with divisive behaviors.

\section{Methods}

\subsection{General structure of the model}

\subsubsection{Five layers}

Figure 3 presents the global architecture of our model. The layered architecture of a retina suggests a model made of successive continuous spatio-temporal maps that progressively transmit, and transform, the incoming signal. The incoming light on the retina is described as $L(x, y, t)$, a quantity that can be measured in $\mathrm{cd} / \mathrm{m}^{2}$ and defined for every spatial point $(x, y)$ of the retina at each time $t$. Similarly, each layer of cells or synaptic interaction between cells is supposed to form a spatial continuum, driven by specific differential equations.

The first stage of our model deals with signal processing done in the Outer Plexiform Layer (OPL). It involves the two first layers of cells in the retina: the light receptors and the horizontal cells. This stage is modeled as a simple spatio-temporal linear filtering, similarly to existing retina models such as [14]. We detail this OPL filter in Section 2.2. When applied to the input sequence $L(x, y, t)$, the OPL filter defines a band-pass excitatory current $I_{\mathrm{OPL}}(x, y, t)$ which is fed to bipolar cells.

The second stage models synaptic interactions in the Inner Plexiform Layer (IPL). It involves two cellular layers: the bipolar cells and the amacrine cells. Biologically, amacrine cells exist under various sub-types with different physiological characteristics and, likely, functionalities [18, 23]. In this second stage of our model, we only focus on two presumed roles of amacrine cells. The first role is to provide contrast gain control on bipolar cells, through a variable feedback leak conductance $g_{\mathrm{Am}}^{L}(x, y, t)$. This interaction is represented by the two small arrows between bipolar cells and amacrine cells in Figure 3. The second role of amacrine cells is to shut down (make more phasic) the response of ganglion cells. The output of the IPL stage is an excitatory current $I_{\text {Gang }}(x, y, t)$ which is fed to ganglion cells.

The third stage of our model is the ganglion cells layer. It is a discrete set of integrate-and-fire cells paving the visual field, and generating spike trains from the input current $I_{\text {Gang. The cells can }}$ 
either be X-type (the blue arrow, representing a one-to-one connection from bipolar cells) or Y-type (the blue cone, representing a synaptic pooling of the excitatory current). See the sequel (especially Section 4.2.1) for further explanations on the different types of ganglion cells. The results in Section 3.4.2 display images reconstructed from these emitted spike trains.

\subsubsection{Horizontal couplings}

When a layer of retinal cells integrates a current from some presynaptic layer of cells, it performs spatio-temporal averaging on its presynaptic input. Temporally, the averaging occurs because of synaptic delays, and mostly because each neuron is a leaky electrical capacitor that functions as a low-pass 'RC' circuit. Spatially, an averaging also occurs, for two anatomical reasons. First reason is the possible dendritic spread of the cells, meaning that each cell integrates a signal from a small patch of presynaptic cells around its location. Second reason is the electrical coupling of each cell with its neighboring cells of the same layer, through gap-junctions, which leads to a local homogenization of neighboring cells' potentials.

The low-passing of a signal every time it is transmitted to a new layer of cells is functionally very important in the retina. In the OPL, it gives rise to the center-surround architecture of retinal filtering. In the IPL, it provides amacrine cells with a local measure of contrast in the image. Our model always uses the same formal kernel $K_{\sigma, \tau}(x, y, t)$ to account for the low-passing of a signal (see Appendix for more justification):

$$
K_{\sigma, \tau}(x, y, t)=G_{\sigma}(x, y) \exp (-t / \tau) / \tau,
$$

if $t>0$, and zero otherwise. $G_{\sigma}(x, y)$ is the two-dimensional normalized Gaussian distribution of standard deviation $\sigma . K_{\sigma, \tau}$ is thus a low-pass filter, separable in time and space, represented in Figure 2. It sums to one, meaning that it is only an averaging filter, without a linear gain. It is null when $t<0$, as all causal filters. Throughout the rest of this article, all temporal filters of the form $\exp (-t / \tau)$ will implicitly be considered null when $t<0$.

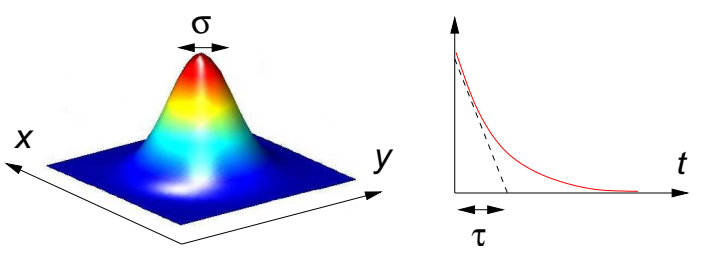

Figure 2: Low-pass coupling kernel $K_{\sigma, \tau}(x, y, t)$ that models signal averaging through a layer of retinal cells.

\subsection{Outer Plexiform Layer}

Physiologically, Outer Plexiform Layer (OPL) is the designation for the first layer of synapses in the retina, which is the locus of synaptic interactions between light receptors, horizontal cells and 


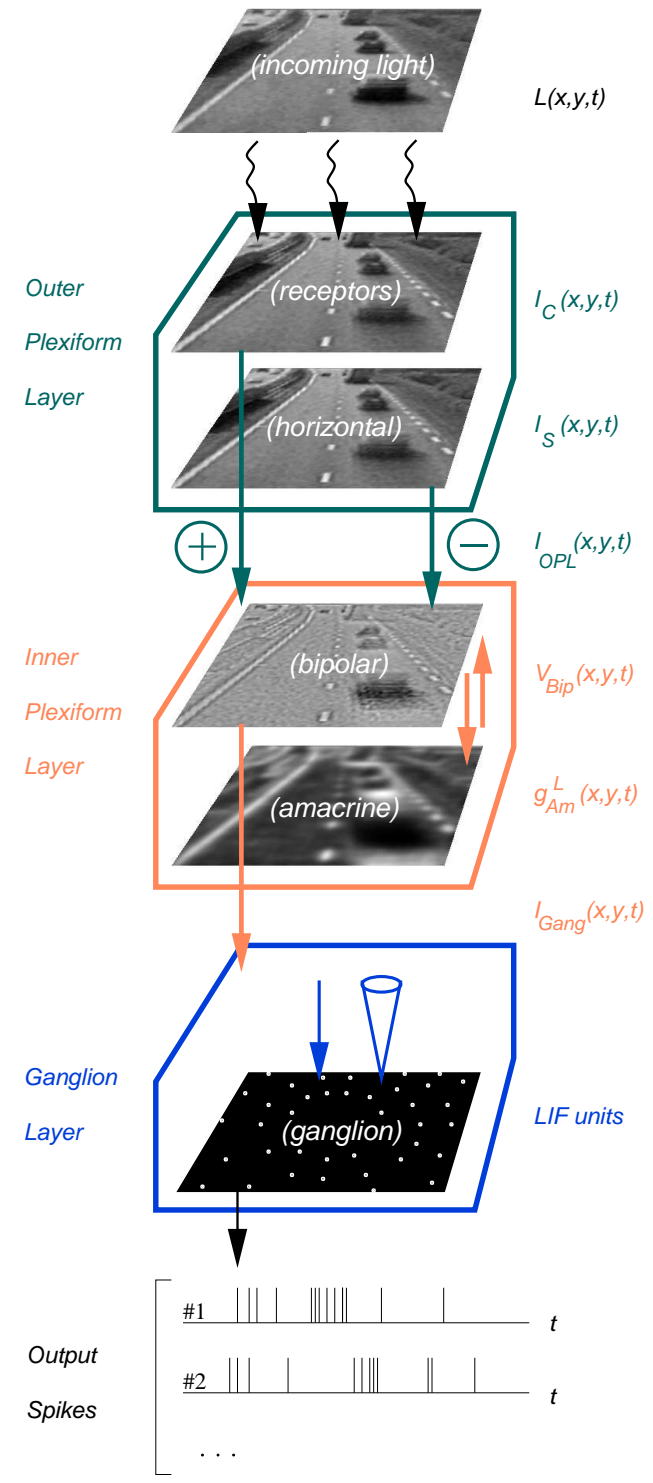

Figure 3: Schematic view of our model, inspired by the five layers of cells in mammalian retinas. On the left hand side, names for the three stages of our model. Main notations are indicated in the right-hand side. Note that except for the last layer (ganglion cells), successive signals are supposed to consist of spatially continuous maps.

$\mathrm{RR} \mathrm{n}^{\circ} 6243$ 
bipolar cells $[23,18]$. We model the processing done in the OPL by a simple linear filter. This filter gives rise to the well-known center-surround architecture of retinal processing, detailed in Section 2.2.1. Furthermore, our model introduces another retinal characteristic at the level of the OPL: slow cellular adaptation to constant stimuli (see Section 2.2.2).

\subsubsection{Center-surround organization in the OPL}

Retinal signals display a spatial opposition between a precise center signal, and a wider surround signal providing each location of the retina with a measure of the average illumination in the neighborhood. As in the Mahowald-Mead retina [21], later extended by Herault [14], we suppose that the center-surround organization arises mostly in the OPL, through competing contributions from light receptors (center, or $C$ ) and horizontal cells (surround, or $S$ ) onto bipolar cells. The resulting current $I_{\mathrm{CS}}(x, y, t)$ received by bipolar cells from light receptors and horizontal cells is thus modeled as

$$
I_{\mathrm{CS}}(x, y, t)=I_{C}(x, y, t)-I_{S}(x, y, t),
$$

with

$$
\begin{gathered}
I_{C}(x, y, t)=\lambda_{\mathrm{OPL}} \quad K_{\sigma_{C}, \tau_{C}} * L \quad(x, y, t), \\
I_{S}(x, y, t)=\lambda_{\mathrm{OPL}} \quad K_{\sigma_{S}, \tau_{S}} * L \quad(x, y, t),
\end{gathered}
$$

where sign $*$ represents spatio-temporal convolution. The constant $\lambda_{\mathrm{OPL}}$ is the overall gain of the center-surround filter, expressed in Hertz per unit of luminance. It is common to the calculations of $I_{C}(x, y, t)$ and $I_{S}(x, y, t) . K_{\sigma_{C}, \tau_{C}}$ and $K_{\sigma_{S}, \tau_{S}}$ are low-pass normalized filters (spatio-temporal integral of one) as presented in equation (1). The surround signal is more low-pass than the center signal, meaning $\sigma_{S}>\sigma_{C}$ and $\tau_{S}>\tau_{C}$. Temporally, this is because horizontal cells receive their input from light receptors, so that their signal develops with one more synapse and one more cellular integration than receptors. Spatially, the very low-pass (averaging) properties of horizontal cells arise from strongly coupling gap junctions with their neighboring horizontal cells [24, 23, 18].

The center-surround filter, as defined by (2)-(4), is a difference of two low-pass filters, which makes it a band-pass filter. Spatially, the center-surround filter can be associated to a classical difference of Gaussians (DOG). Temporally, it is biphasic. As a result, bipolar cells act as the same time as edge detectors and movement detectors.

However, note that the center-surround filter is not separable in time and space: it cannot be written as the product of a spatial kernel by a temporal kernel. As a result, it can be considered neither temporally low-pass (or tonic, in biological terminology) nor temporally high-pass (or phasic). Its temporal properties depend on the spatial structure of the image at each location. On image edges, the filter is tonic, meaning that an edge in the image always induces a long-lasting response of the filter. On the contrary, on uniform zones of the image, the filter is phasic, meaning that it only detects movement or changes in luminosity, and has null response otherwise.

This filter is able to detect luminosity changes, even in a spatially uniform zone. This would not be the case for a separable filter, like a spatial DOG multiplied by a temporal difference-ofExponentials. Indeed, the response of such a separable filter on a uniform region would always be zero because of the DOG properties, even if the luminosity does vary in time. 


\subsubsection{Slow cellular adaptation}

Cellular adaptation on 'medium-slow' time scales, in the order of a second, is observed in the majority of cortical neurons [3, 7], as well as in the retina (see experiments in Section 3.3). Whenever a retinal cell is presented with a temporally constant stimulus, its response progressively diminishes, even for those retinal cells that are termed tonic because of their relatively long-lasting response. This adaptation to constant illumination starts as early as in light receptors, that display a complicated non-linear adaptation to light levels, within the order of a second [27, 30].

Our model takes this adaptation into account through a simple temporal linear filter, that we suppose to be active from the very beginning of retinal processing, at the level of light receptors themselves. Since the signal of light receptors is at the source of all subsequent retinal processing, the effects of adaptation will naturally be transmitted through all the layers of the model.

Computationally, adaptation is added as a supplementary high-pass filter, with a time constant of a few hundreds of milliseconds, to generate the final output of our OPL stage:

$$
I_{\mathrm{OPL}}(x, y, t)=K^{\text {adap }} \stackrel{t}{*} I_{\mathrm{CS}}(x, y, t),
$$

where $\stackrel{t}{*}$ denotes temporal convolution, and $K^{a d a p}(t)$ is a partially high-pass temporal filter defined by

$$
K^{\text {adap }}(t)=\delta_{0}(t)-w^{a d a p} \exp \left(-t / \tau^{a d a p}\right) / \tau^{a d a p},
$$

where $\delta_{0}(t)$ is a Dirac function, representing the original signal. $w^{\text {adap }}$ is a constant between 0 and 1 giving the relative strength of the adaptation effect. It was chosen to fit experimental data in Section 3, with typical values of around 0.5 , a value compatible with measurements of light-evoked responses in retinal cones [27]. $\tau^{a d a p}$ is the temporal scale of the cellular adaptation, typically around $200 \mathrm{~ms}$.

Note that equations (2)-(6) can all be grouped in a single kernel $K_{\mathrm{OPL}}(x, y, t)$ that directly encompasses the linear filtering from input light $L(x, y, t)$ to a current $I_{\mathrm{OPL}}(x, y, t)$ onto bipolar cells, including the effects of light adaptation. It writes, functionally:

$$
I_{\mathrm{OPL}}(x, y, t)=K_{\mathrm{OPL}} * L \quad(x, y, t)
$$

with

$$
K_{\mathrm{OPL}}=\lambda_{\mathrm{OPL}} K^{\text {adap }} \stackrel{t}{*}\left(K_{\sigma_{C}, \tau_{C}}-K_{\sigma_{S}, \tau_{S}}\right) .
$$

This makes $K_{\mathrm{OPL}}$ the non-separable difference of a center kernel with a surround kernel, each of these two kernels being the separable product of a spatial Gaussian with a partially transient temporal filter. This is actually the type of linear approximation that was found to account best for the response of cells in the LGN, by Cai et al [2].

\subsection{Inner Plexiform Layer}

Physiologically, the Inner Plexiform Layer (IPL) is the second layer of synapses in the retina. It is the locus of synaptic interactions between bipolar cells, amacrine cells, and ganglion cells (the output 
cells of the retina). Amongst other functionalities, synaptic interactions in the IPL are supposed to be at the origin of contrast gain control [28,32,9]. We present in Section 2.3.1 a model of contrast gain control based on a non-linear feedback between a certain class of amacrine cells and bipolar cells. Then, Section 2.3.2 presents how to transform the potential of bipolar cells into an excitatory current (from which spike trains will be generated), thanks a temporal high-passing and a smooth synaptic rectification.

\subsubsection{Bipolar charging equation and the contrast gain control}

Let us start by describing the equations of our bipolar/amacrine feedback loop. Cellular integration in bipolar cells, defined by their membrane potential $V_{\text {Bip }}(x, y, t)$, includes synaptic inputs from the OPL but also a feedback shunting conductance from amacrine cells denoted by $g_{\mathrm{Am}}^{L}(x, y, t)$. The system is

$$
\begin{aligned}
\frac{d V_{\mathrm{Bip}}}{d t}(x, y, t) & =I_{\mathrm{OPL}}(x, y, t)-g_{\mathrm{Am}}^{L}(x, y, t) V_{\mathrm{Bip}}(x, y, t) \\
g_{\mathrm{Am}}^{L}(x, y, t) & =K_{\sigma_{\mathrm{Am}}, \tau_{\mathrm{Am}}} * T_{\mathrm{BA}}\left(V_{\mathrm{Bip}}\right)(x, y, t) .
\end{aligned}
$$

Here $g_{\mathrm{Am}}^{L}(x, y, t)$ represents the summed effects of all leak conductances in the bipolar cell layer (including inert leaks). In our models, this total leak depends on the activity of some amacrine cells. $K_{\sigma_{\mathrm{Am}}, \tau_{\mathrm{Am}}}(x, y, t)$ is a coupling low-pass filter as in (1), corresponding to cellular integration in amacrine cells, and $T_{\mathrm{BA}}\left(V_{\mathrm{Bip}}(x, y, t)\right)$ is the synaptic current induced by bipolar cells in amacrine cells. For reasons that we discuss in the next paragraph, we choose the transmission function $T_{\mathrm{BA}}$ to be:

$$
T_{\mathrm{BA}}\left(V_{\mathrm{Bip}}\right)=g_{\mathrm{BA}}+\lambda_{\mathrm{BA}} V_{\mathrm{Bip}}^{2} .
$$

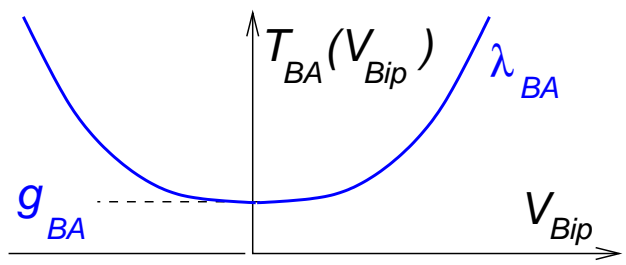

Figure 4: Synaptic activation function $T_{\mathrm{BA}}\left(V_{\mathrm{Bip}}\right)$ from Bipolar layer to Amacrine layer.

Now, let us comment the model (9)-(11). First, from (9), it appears that amacrine cells have a divisive effect on the values of $V_{\mathrm{Bip}}$ : by changing the leak in the membrane of bipolar cells, they influence the gain of the current integration (see discussion in Section 4). Second, the whole system works as a feedback loop, since amacrine cells, that control the gain of integration in bipolar cells, depend themselves on the level of activity of bipolar cells, through equation (10). Through the symmetric shape of the transmission function $T_{\mathrm{BA}}$ in (11), amacrine cells activity depends only on 
the absolute value of $V_{\mathrm{Bip}}$. Furthermore, the convex shape of $T_{\mathrm{BA}}$ implies different behaviors of the system, depending on the contrast:

- At small contrasts, the system has a quasi-linear working range. Indeed, when the input current $I_{\mathrm{OPL}}(x, y, t)$ has small variations, it translates into small variations of the bipolar potential, so that $V_{\mathrm{Bip}}(x, y, t)$ remains in the 'central region' of function $T_{\mathrm{BA}}$, where $V_{\mathrm{Bip}}(x, y, t)^{2} \simeq 0$. As a result, $g_{\mathrm{BA}}$ remains the principal, constant, leaking force in equation (9), and integration remains quasi-linear at the level of bipolar cells.

- At high contrasts, by opposition, as $\left|V_{\mathrm{Bip}}(x, y, t)\right|$ enters the 'big value' range of function $T_{\mathrm{BA}}$, amacrine cells become truly active and modulate significantly the leakage term in equation (9). As a result, bipolar cells start responding sub-linearly to the input current $I_{\mathrm{OPL}}(x, y, t)$.

The precise choice of function $T_{\mathrm{BA}}\left(V_{\mathrm{Bip}}\right)$ is arbitrary in our model. The only important constraint for our model to reproduce experimental curves, was that function $T_{\mathrm{BA}}$ be strictly convex; it allowed enhancement of the contrast gain control effect, according to the intuitive explanation above.

The model (9)-(11) has several parameters with different roles. Before commenting on these parameters, let us note that for simplicity reasons, equation (9) has been reduced dimensionally. $V_{\mathrm{Bip}}$ is dimensionless, while $I_{\mathrm{OPL}}$ and $g_{\mathrm{Am}}^{L}$ have the dimensions of frequencies and are thus expressed in Hertz. Thanks to this reduction, there is no more membrane capacity factor on the left side of equation (9).

In (11), parameter $g_{\mathrm{BA}}$ represents the inert leaks in bipolar cells' membrane (because filter $K_{\sigma_{\mathrm{Am}}, \tau_{\mathrm{Am}}}$ has a gain of 1). It does not depend on the mean level of activation $V_{\mathrm{Bip}}^{2}$, as relayed by amacrine cells. On the contrary, $\lambda_{\mathrm{BA}}$ in (11) is the gain of amacrine cells' dynamic contribution to the leaks. It is also expressed in Hertz. It fixes the overall strength of the gain control feedback loop.

In equation (10), $\sigma_{\mathrm{Am}}$ is the typical size of the spatial coupling in amacrine cells. It corresponds to the field of influence of one bipolar cell on its post-synaptic amacrine cells, measured in terms of retinal distance. Finally, $\tau_{\mathrm{Am}}$ in $(10)$ is the delay due to synaptic transmission and cellular integration in amacrine cells. Due to the hypothetical nature of this model stage, there is no data to directly fix parameters $\sigma_{\mathrm{Am}}$ and $\tau_{\mathrm{Am}}$, but simple extrapolation is feasible; $\sigma_{\mathrm{Am}}$ can be fixed with similar values as $\sigma_{S}$ chosen to generate the surround signal in horizontal cells in equation (4), while $\tau_{\mathrm{Am}}$ takes the typical values for cellular integration in any of the retinal layers. In the experiments presented herein (Section 3), $\sigma_{\mathrm{Am}}$ and $\tau_{\mathrm{Am}}$ were found to have only a moderate influence on the system, as long as they remained in a 'biological' range. However, precise mathematical study of these parameters' influence on the dynamical system (9)-(11) is yet to be done (see discussion in Section 4.1).

Finally, remark that bipolar cells react symmetrically with positive and negative contrasts. A signal in the range $V_{\mathrm{Bip}}<0$ is our functional equivalent to 'OFF' sensitive bipolar cells in a real retina. So in our model, since function $T_{\mathrm{BA}}$ in (11) is even, amacrine cells receive input from 'ON' as well as from 'OFF' bipolar cells. In real retinas, this contrast symmetry could be achieved either through a single class of 'ON and OFF' sensitive amacrine cells, or through two classes of amacrine cells (one 'ON' and one 'OFF') acting simultaneously on 'ON' and 'OFF' bipolar cells. Both mechanisms can be reduced to the model proposed here. 


\subsubsection{Synaptic current upon ganglion cells}

Second part of the IPL in our model is to convert the bipolar potential $V_{\mathrm{Bip}}(x, y, t)$ into an excitatory post-synaptic current $I_{\text {Gang }}(x, y, t)$ for ganglion cells, that will generate spike trains. This conversion implies two stages: The first is a high-pass temporal filter that sums up the effect of different transients in the IPL. The second is a smooth synaptic rectification to obtain a positive excitatory current $I_{\text {Gang. This can be written as follows }}$

$$
\begin{gathered}
V_{\mathrm{Bip}}^{\text {trs }}(x, y, t)=K^{t r s}{ }^{t} V_{\mathrm{Bip}}(x, y, t), \\
I_{\mathrm{Gang}}(x, y, t)=T_{\mathrm{BG}}\left(V_{\mathrm{Bip}}^{\text {trs }}\right)(x, y, t),
\end{gathered}
$$

where $\stackrel{t}{*}$ denotes temporal convolution, and $K^{\operatorname{trs}}(t)$ ( $\operatorname{trs}$ standing for transient) is a partially highpass temporal filter, defined exactly as the OPL adaptation filter $K^{a d a p}$ in (6):

$$
K^{t r s}(t)=\delta_{0}(t)-w^{t r s} \exp \left(-t / \tau^{t r s}\right) / \tau^{t r s},
$$

where $\delta_{0}(t)$ is a Dirac function, representing the original signal, and $w^{\text {trs }}$ is a constant between 0 and 1. The typical values for $w^{\text {trs }}$ will depend on the type of ganglion cell modeled, as detailed in the sequel. $\tau^{\text {trs }}$ is the temporal scale of the transient, typically a few tens of milliseconds. Finally, the transmission function $T_{\mathrm{BG}}$ in (13) is a smooth synaptic rectification defined by (see Figure 5):

$$
T_{\mathrm{BG}}(v)= \begin{cases}\frac{T_{0}^{2}}{T_{0}-\lambda_{\mathrm{BG}}\left(v-V_{\mathrm{BG}}\right)} & \text { if } v<V_{\mathrm{BG}}, \\ T_{0}+\lambda_{B G}\left(v-V_{\mathrm{BG}}\right) & \text { if } v>V_{\mathrm{BG}},\end{cases}
$$

where $\lambda_{B G}$ and $T_{0}$ have, again, the dimension of 'reduced currents' expressed in Hertz. $V_{\mathrm{BG}}$ is the 'linearity threshold' of the cell, i.e. the value after which transmission becomes linear. Note that $T_{\mathrm{BG}}\left(V_{\mathrm{BG}}\right)=T_{0}$.

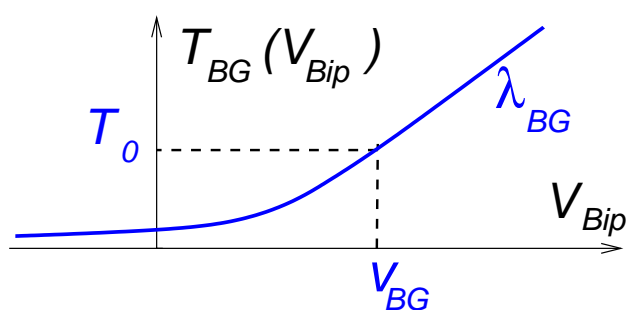

Figure 5: Synaptic activation function $T_{\mathrm{BG}}\left(V_{\mathrm{Bip}}\right)$ from Bipolar layer to Ganglion layer.

Now, let us comment the model (12)-(13). Equation (12) is the location in our model where the balance can be fixed between phasic and tonic properties of the cells one wishes to model, by playing on parameter $w^{t r s}$. The equation creates a 'secondary' bipolar signal $V_{\mathrm{Bip}}^{\text {trs }}(x, y, t)$ with additional high-pass properties on the time scale of tens of milliseconds. This is typically the order 
of the time constant for the response of phasic ganglion cells. By taking $w^{\text {trs }}=1$, one obtains a cell whose response is totally phasic, meaning that the response to a constant stimulation shuts down after one or two hundreds of milliseconds (total length of the burst should not be confused with the time constant for the burst).

In the primate retina, Parasol cells are known to be totally phasic, by opposition to Midget cells that are considered tonic. In the cat retina, Y cells are phasic, and X cells tonic [10] (see Section 4.2.1). To reproduce experimental results on cat ganglion cells (Sections 3.2 and 3.3), we used typical values of $w^{t r s}=1$ for $\mathrm{Y}$ cells, and of $w^{\text {trs }}=0.7$ for $\mathrm{X}$ cells. This last value proves the existence of an intrinsic transient even in 'tonic' cells, a result that is best visible in the Bode diagrams for these cells (Section 3.2).

There are many plausible biological explanations for the transient properties intrinsic to all ganglion cells. Likely, the main reason is the existence of specific amacrine cells in the IPL that were found to cut the responses of ganglion cells $[25,23,18]$, whether through a feedback to bipolar cells, whether by direct inhibition on ganglion cells. See discussion in Section 4.1.

To conclude, equation (13) simply describes a synaptic rectification, which is a very common feature in neural modeling and in retinal models $[4,5,12]$. A smooth rectification is needed here to account for the observed 'low-input' range of ganglion cells, where ganglion cells clearly act underlinearly, but are not totally silent either (see cell simulations in Section 3.3).

Note that if one wishes to simulate 'OFF' ganglion cells, equation (13) must display an inversion of sign, thus becoming

$$
I_{\text {Gang }}(x, y, t)=T_{\mathrm{BG}}\left(-V_{\mathrm{Bip}}^{\text {trs }}\right)(x, y, t) .
$$

\subsection{Ganglion layer}

The last stage of our model is the generation of spike trains, obtained from the excitatory current $I_{\text {Gang }}(x, y, t)$. Each ganglion cell will be represented by a spiking Leaky-Integrate-and-Fire neuron (LIF), described in Section 2.4.1. Each cell will generate its spiking output by integrating in time the current $I_{\text {Gang }}(x, y, t)$, with a last synaptic pooling in the case of cat $\mathrm{Y}$ cells (and primate Parasol cells). Then, Section 2.4.2 shows how to define a whole retina as an assembly of LIF. Two possible retinal organizations are discussed, log-polar or homogeneous.

\subsubsection{Spike generation in ganglion cells}

Let us consider $N$ ganglion cells $C_{n}(n=1 \ldots N)$ paving the retinal space (see Section 2.4.2 for their repartition and parameters) and let us denote by $V_{n}$ the potential of cell number $n(n=1 \ldots N)$ centered at position $\left(x_{n}, y_{n}\right)$. 
Then, if $C_{n}$ is a cat $\mathrm{X}$ cell (or primate Midget cell), we generate its spiking output with a standard LIF model:

$$
\left\{\begin{array}{l}
\frac{d V_{n}}{d t}=I_{\text {Gang }}\left(x_{n}, y_{n}, t\right)-g^{L} V_{n}(t)+\eta_{v}(t), \\
\text { Spike when threshold is reached: } V_{n}\left(t_{\mathrm{spk}}\right)=1, \\
\text { Refractory period: } V_{n}(t)=0 \text { while } t<t_{\mathrm{spk}}+\eta_{\mathrm{refr}}, \\
\text { and (16) again, }
\end{array}\right.
$$

where $\eta_{v}(t)$ and $\eta_{\text {refr }}$ are two noise sources that can be added to the spike generation process in order to reproduce the trial-to-trial variability of real ganglion cells, following the experimental results of Keat et al [17]. $\eta_{v}(t)$ is taken as a Brownian movement that has the dimension of a current. Integration of this current through equation (16) is equivalent to adding to $V_{n}(t)$ a Gaussian auto-correlated process with time constant $1 / g^{L}$ (typically, $20 \mathrm{~ms}$ ), and variance $\sigma_{v}$. The amplitude of $\eta_{v}(t)$ is chosen for $\sigma_{v}$ to be around 0.1. $\eta_{\text {refr }}$ is a stochastic absolute refractory period that is randomly chosen after each spike, following a normal law, typically $\mathcal{N}(3 \mathrm{~ms}, 1 \mathrm{~ms})$.

Modeling cat $\mathrm{Y}$ cells (and primate Parasol cells) requires an additional feature to account for the wide dendritic tree of these cells. Equation (16) becomes

$$
\frac{d V_{n}}{d t}=\left(G_{\sigma_{\text {Gang }}} \stackrel{x, y}{*} I_{\text {Gang }}\right)\left(x_{n}, y_{n}, t\right)-g^{L} V_{n}(t)+\eta_{v}(t),
$$

where $G_{\sigma}(x, y)$ is again the normalized Gaussian of standard deviation $\sigma$. Indeed, Y-type cells possess a spatial non-linearity, as observed by [10]. The simplest explanation of this spatial non-linearity is a spatial pooling that would occur after the synaptic rectification onto these ganglion cells. This explanation, first proposed by Hochstein and Shapley [15], is at the base of the Freeman and EnrothCugell model for Y-type cells [9]. Furthermore, the biological basis was justified experimentally $[8,18]$ : The spread of the dendritic tree of Y-type cells is large enough to significantly average the synaptic input from bipolar cells over a consequent spatial extent.

\subsubsection{Ganglion cell sampling configurations}

The whole model presented above holds when modeling a small region of the retina, in which the density of retinal cells can be considered uniform. In that case, all filtering scales and parameters are constant and do not depend on the spatial position of each cell. Our simulation software can easily handle such a uniform distribution of cells. And in most mammalian species, the whole retina has a fairly uniform density of cells.

However, the primate retina taken as a whole is not uniform at all. Density of cells and filtering scales depend on the position considered in the retina. One needs to distinguish the fovea in the center, from the surround of the visual field where precision is less. A simple way is to define a scaling function, that describes at the same time the local density of cells and the spatial scales of filtering in the different regions of the retina. 
Our model can be implemented using a radial and isotropic density function that depends on the distance $r$ from the center of the retina. We define a one-dimensional log-polar density function $d(r)$ as

$$
d(r)= \begin{cases}d_{0} & \text { if } r<R_{0}, \\ d_{0} /\left(1+K\left(r-R_{0}\right)\right) & \text { if } r>R_{0},\end{cases}
$$

where $R_{0}$ is the size of the fovea and $K$ is the speed of density decrease outside of the fovea. When $K=1 / R_{0}$, this amounts to a traditional log-polar sampling scheme. The density of cells in a given region of the retina at eccentricity $r$ will then be given by $d(r)^{2}$, while all spatial filtering scales of the model presented before $\left(\sigma_{C}, \sigma_{S}, \sigma_{\mathrm{Am}}, \sigma_{\mathrm{Gang}}\right)$ scale with $d(r)^{-1}$.

The choice of such a density function is biologically justified: Receptive fields for ganglion cells have experimentally been found to scale with a negative power of $r$, between $r^{-0.7}$ and $r^{-1}$ according to the type of cell [6].

Let us now present our results. After a short presentation of the simulator Virtual Retina that can implement our model on a large scale, we validate the model by reproducing physiological recordings on single ganglion cells. Finally, a large-scale simulation demonstrates the perceptual interest of the present retina model.

\section{Results}

\subsection{Retina simulator customization}

The software Virtual Retina ${ }^{1}$ is documented and download-able at the following address:

http://www-sop.inria.fr/odyssee/team/Adrien.Wohrer/retina

It implements the model presented in this article, with the following characteristics:

Possibility of large-scale simulations: Up to 100,000 spiking cells can be simulated in a reasonable time (speed of around 1/100 real time).

$X M L$ definition file: All parameters for the different stages of the model are defined in a single customizable XML file.

Two possible density functions: The software provides two customized sampling schemes to pave the visual field. First option is a uniform, square array of cells, to precisely model a small region of the retina in which cell density can be considered uniform. In that case, all spatial filtering scales of the model $\left(\sigma_{C}, \sigma_{S}, \sigma_{\mathrm{Am}}, \sigma_{\mathrm{Gang}}\right)$ are constant throughout the whole image, and the corresponding Gaussian filters $G_{\sigma}(x, y)$ are implemented thanks to traditional recursive Deriche filters. Second option is a circular sampling (ganglion cells are located along concentric circles), following a nonuniform density $d(r)$ as defined by equation (18). This can be used for coarse simulations of the whole visual field of a primate. In that case, as explained in Section 2.4.2, the spatial filtering scales of the model ( $\left.\sigma_{C}, \sigma_{S}, \sigma_{\mathrm{Am}}, \sigma_{\mathrm{Gang}}\right)$ also change according to the distance $r$ from the center of the fovea, proportionally to $d(r)^{-1}$. However, the corresponding Gaussian filters $G_{\sigma}(x, y)$ can still be

\footnotetext{
${ }^{1}$ This software is in course of being referenced under IDDN (Inter Deposit Digital Number) certifi cation.
} 
implemented recursively (implying a significant gain in computational speed), thanks to a recursive filtering with inhomogeneous recursive coefficients, as proposed in [29].

Fixation microsaccades: Finally, the software allows to include a simple random microsaccades generator at the input of the retina, to account for fixation eye movements, as inspired from [22].

\subsection{Multi-sinus experiment and the contrast gain control effect}

Let us now validate our retina model, through comparisons with intra-cellular recordings on real cat ganglion cells. We start by a reproduction of the Shapley and Victor [28] multi-sinus experiment that gave the first quantitative measures of contrast gain control in the retina. Good reproduction of these results makes our model a plausible interpretation of the gain control taking place in the retina. Their experimental protocol is explained in Section 3.2.1, the experimental results are commented in Section 3.2.2. In Section 3.2.3 we present the performances of our model.

\subsubsection{Experimental protocol}

Original experiment of Shapley and Victor [28] was pursued on an ON-center X-type ganglion cell of an anesthetized cat. Input stimulus $L(x, y, t)$ to the cell was a static spatial sinusoidal grating of fixed mean luminance $\bar{L}=20 \mathrm{~cd} / \mathrm{m}^{2}$, temporally modulated by a sum of sinusoids with varying contrast:

$$
L(x, y, t)=\bar{L}\left(1-c \operatorname{Gr}(x, y) \sum_{i=1}^{8} \sin \left(\xi_{i} t\right)\right),
$$

where $\operatorname{Gr}(x, y)$ is a spatial grating function with normalized amplitude (between -1 and 1 ). The $\xi_{i}$ are a set of eight temporal frequencies that logarithmically span the frequency range from about $0.2 \mathrm{~Hz}$ to $32 \mathrm{~Hz}$. $c$ was a measure for the global level of contrast of the experiment, and was doubled between each two successive experiments. A sample of the corresponding temporal course is presented in Figure 6. Response of the ganglion cell was measured as a trial-averaged firing rate. For each input contrast $c$, the cell's output firing rate was Fourier-analyzed at each of the input frequencies $\xi_{i}$, thus yielding a set of eight amplitudes and eight phases. This set provides a measure for the linear kernel (first-order Wiener kernel) that best fits the cell's response, for the given experiment at given contrast $c$.

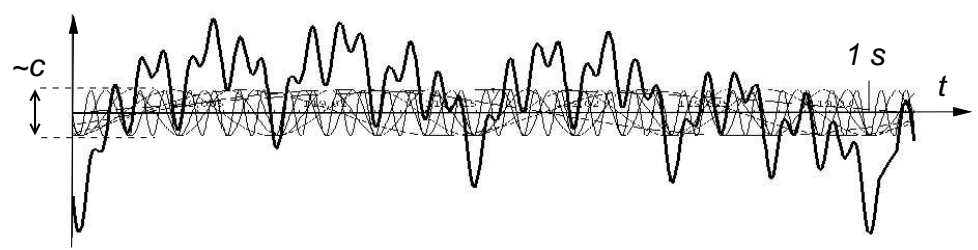

Figure 6: Temporal course over one second of the multi-sinus stimulus (19) and of its sinusoidal components. The amplitude of each sinusoidal component is proportional to contrast $c$. 


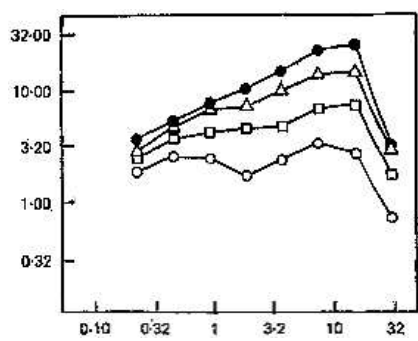

(a) $\log$ Amplitude

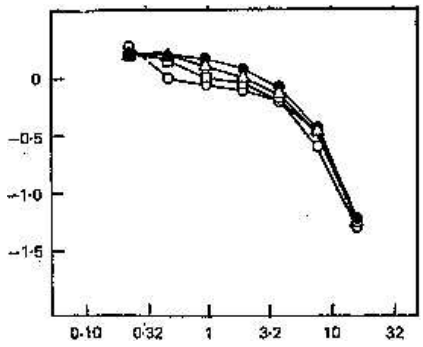

(b) Phase

Figure 7: Measurements of a cat ON-center X ganglion cell, as a function of contrast $c$ [28]. The $\mathrm{X}$-axis corresponds to the temporal frequency $(\mathrm{Hz})$ of the input signal $(\xi)$. Figure (a) shows the amplitude (impulses/sec), and (b) the phase (rad) of the first-order frequency kernel. The different curves are obtained for different values of contrasts for the input signal. From lower curve to upper curve, $c$ was $0.0125,0.025,0.05,0.1$.

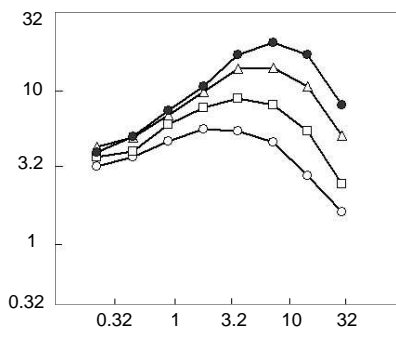

(a) $\log$ Amplitude

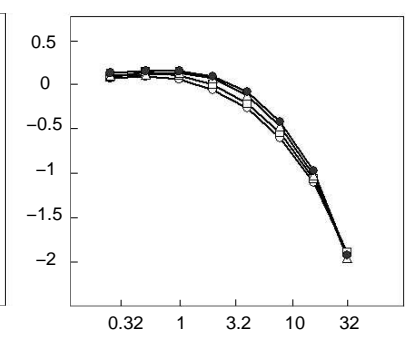

(b) Phase

Figure 8: Temporal frequency kernels at different levels of contrast reproduced with our retina model. Test grating: Normalized mean luminance of $0.5,0.2$ cycles/deg. OPL parameters: $\sigma_{C}=0.88 \mathrm{deg}, \sigma_{S}=2.35 \mathrm{deg}, \tau_{C}=10 \mathrm{~ms}, \tau_{S}=20 \mathrm{~ms}, \lambda_{\mathrm{OPL}}=150 \mathrm{~Hz}$ per luminance unit. $w^{\text {adap }}=0.5, \tau^{\text {adap }}=200 \mathrm{~ms}$. Gain control parameters: $g_{\mathrm{BA}}=5 \mathrm{~Hz}, \lambda_{\mathrm{BA}}=100 \mathrm{~Hz}, \tau_{\mathrm{Am}}=20$ $\mathrm{ms}, \sigma_{\mathrm{Am}}=2.5 \mathrm{deg}$. Spike generation parameters: $\lambda_{\mathrm{BG}}=100 \mathrm{~Hz}, w^{\text {trs }}=0.7, \tau^{\text {trs }}=30 \mathrm{~ms}$, $V_{\mathrm{BG}}=0, T_{0}=80 \mathrm{~Hz}, g^{L}=50 \mathrm{~Hz}$.

\subsubsection{Experimental observation}

Figure 7 (a) plots the frequency-versus-amplitude data on a log-log scale, with one different curve for each value of contrast $c$. Figure 7 (b) plots the frequency-versus-phase data on a semi-log scale, with one curve for each level of contrast. This representation is that of Bode diagrams in signal theory.

If the ganglion cell really was a linear transducer on the visual input, the modulations in its response would simply be proportional to $c$, meaning that when contrast is doubled, the cell's response would be similar but with doubled amplitude. The phase of the response would not depend on $c$, 
but only on the nature of the linear filter, and all curves in Figure 7 (b) would superimpose. As for amplitude curves, in the log-amplitude representation of Figure 7 (a), they would all be parallel, with each two successive curves being spaced by an interval of length $\log (2)$ as $c$ is doubled.

Thus, Figure 7 reveals deviations from linearity in the cell's behavior. From the amplitude point of view, a compression of the response can be observed at low frequencies, since successive curves are spaced by less than $\log (2)$. From the phase point of view, strong contrasts induce a phase-advance of the response (phase curve shifted upwards), meaning that the answer of the cell is faster at high contrasts. As noticed by [28] and developed more thoroughly in [32], both observed non-linearities (sub-linearity with contrast at low frequencies and phase advance with contrast) could result from the same origin, a filtering stage in the retina whose time constant would depend on the local level of contrast of the image. That is precisely what is provided by our amacrine feedback on bipolar cells.

\subsubsection{Simulation with our model}

The results for our model are displayed in Figure 8. The model reproduces the typical time advance of ganglion responses at high contrasts, displayed in the phase Figure 8 (b). This is because conductance $g_{\mathrm{Am}}^{L}$ in equation (9) determines the time constant of the response of bipolar cells, and that the mean level of $g_{\mathrm{Am}}^{L}$, dependent on the average of $V_{\mathrm{Bip}}^{2}$, is a growing value of contrast. Similarly, the under-linearity of response amplitudes with contrast is also observed in the amplitude Figure 8 (a), where successive curves are separated by less than $\log (2)$. This is because $g_{\mathrm{Am}}^{L}$ in equation (9) increases the leak in the bipolar membrane, and thus lowers the linear gain of bipolar transmission in the case of high input contrast.

Our model also displays the change in shape of frequency kernels, that is typical of contrast gain control. Two elements of the model were found mandatory to correctly reproduce the kernels. First element is the additional temporal transient on ganglion cells, equation (12). This stage of the model is responsible for most of the high-pass behavior observed in Figure 8. For our results to fit the experimental recordings (on a cat X-type cell), the strength of the transient was fixed at about $w^{\text {trs }}=0.7$. This means that even cells known as tonic display some intrinsic high-passing of the signal. Second element is the amacrine shunting feedback (9)-(11), and more especially the shape of function $T_{\mathrm{BA}}$ in equation (11). It must be set strictly convex, so that amacrine feedback becomes significant only at high contrasts, thus increasing the change in shape of the frequency kernel between low and high contrast.

\subsection{Response of cat $\mathrm{X}$ and $\mathrm{Y}$ cells to grating apparitions}

Grating apparitions, or sometimes grating reversals, are a classical stimulus when experimenting on the low-level visual pathway. We reproduce here one of the first recordings of that kind, made on cat ganglion cells in 1966 by Enroth-Cugell and Robson [10]. Their seminal experiments led to the distinction made between two major types of retinal cells in the cat retina, namely $X$ and $Y$ type cells. $X$ type cells were found to be the most accurate spatially (meaning small receptive fields), and to have a rather tonic response to long-lasting stimuli. Y type cells, on the contrary, have a more phasic response, less spatial acuity, and a very typical spatial non-linearity. We briefly remind their results in Section 3.3.1, and those of our model in Section 3.3.2. 

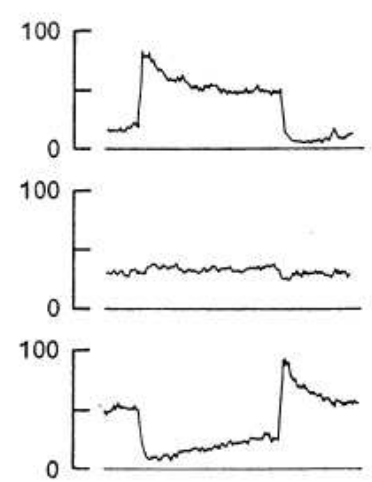

100

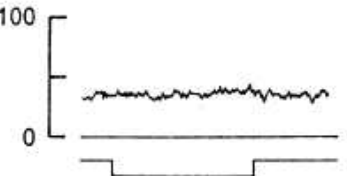

A

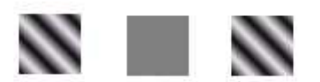

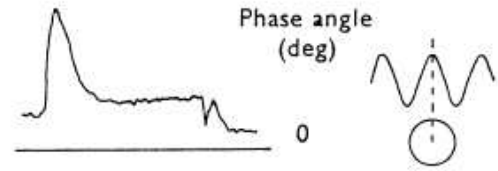
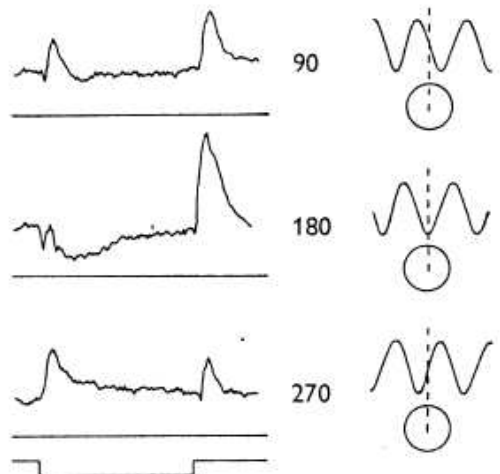

B

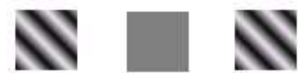

Figure 9: Measurements of cat OFF-center ganglion cells, in response to the disappearance and reappearance of a sinusoidal grating with different spatial offsets [10]. Mean luminance $16 \mathrm{~cd} / \mathrm{m}^{2}$, grating contrast of 0.32. Left: typical X-type ganglion cell. Grating spatial frequency of $0.13 \mathrm{deg}^{-1}$. Right: typical Y-type ganglion cell. Grating spatial frequency of $0.16 \mathrm{deg}^{-1}$.

\subsubsection{Experimental protocol and observations}

The stimulus was a static grating presented to cat OFF-type ganglion cells, that suddenly disappeared, being replaced by a uniform screen of the same mean luminance, and that reappeared after a second. The spatial phase of the grating in front of the cell was varied, so as to test the summing properties of the cells' receptive fields. Grating luminance, contrast and spatial frequency had classical values, detailed in the legend of Figure 9. The spike densities were calculated by averaging spike trains over several trials, and convolving the result with an exponential kernel with a $20 \mathrm{~ms}$ time constant. For each tested cell, the authors also provided a measure of the center and surround receptive fields for the cell. Typical responses for an X cell and a Y cell are presented in Figure 9.

As commented by the authors, the experiment reveals that $X$ cells have a relatively tonic behavior, since their response to a static stimulus lasts for a long time, whereas Y cells are totally phasic, only responding for a few hundreds of milliseconds after stimulus onset, and returning to silent. The experiment is also an illustration of the 'null position' test: For X cells, a spatial phase exists for which the cell has roughly no response to the grating (here, 90 and $270 \mathrm{deg}$ ), when the 'positive' and 'negative' parts of the grating exactly compensate one another thanks to linear summation. For Y 


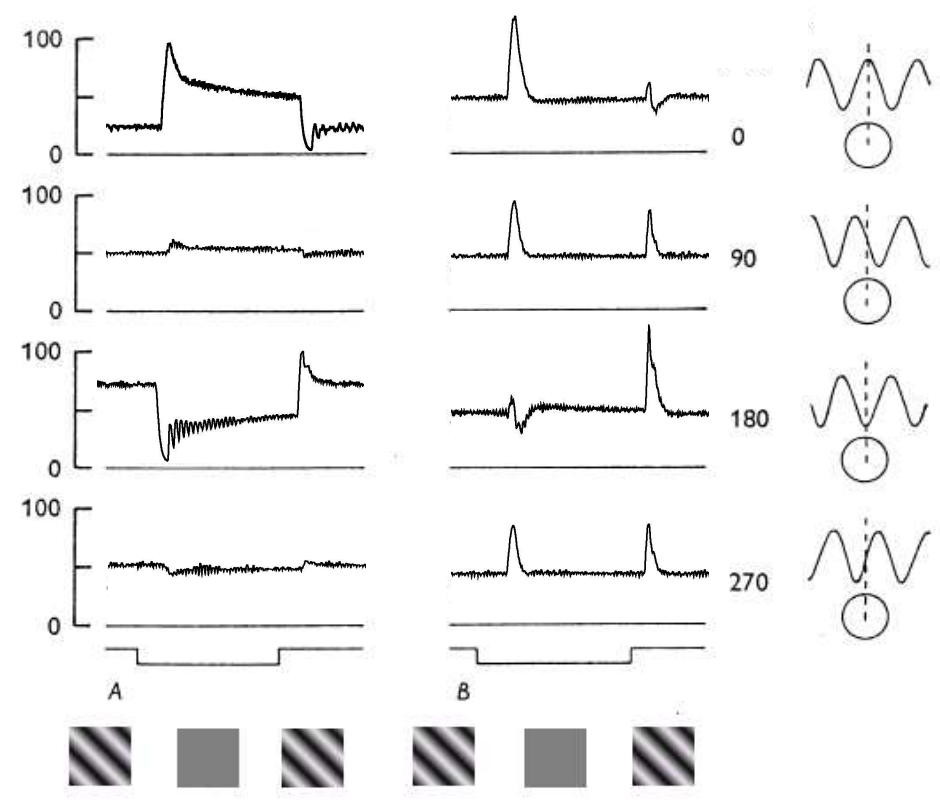

Figure 10: Reproduction of the experiments in Figure 9 by our retina model. Spike trains generated over 80 trials with noise in the spike generation. Firing rates reconstructed with the same method as in the original recordings of [10]. Normalized mean luminance of 0.5 , contrast 0.32 , spatial frequency of $0.13 \mathrm{deg}^{-1}$. Left: X Cell model. Same parameters as the model X-cell used for Figure 8. Right: Y cell model. Same parameters as the model X-cell used for Figure 8, except for the following ganglion cell parameters: $w^{\text {trs }}=1, \sigma_{\mathrm{Gang}}=1.8 \mathrm{deg}, \lambda_{\mathrm{BG}}=400 \mathrm{~Hz}$.

cells such a position does not exist, revealing a spatial non-linearity, as explained already in Section 2.4.1.

Let us add a final remark about the X cell curves in Figure 9 (A). During the 'no grating' period of the stimulation (at the middle), the cell sees exactly the same stimulus in all four experiments, that is a uniform screen. However, even after a whole second of 'no grating' stimulation, the reconstructed firing rates are slightly different according to the phase of the experiment considered! We claim that this is due to a slow adaptation of the cell to its own level of response during the preceding period, when the grating was on. For example, in the ' 180 deg' experiment, the cell has been adapted to a strong response stimulus during the preceding period, and thus it has lowered its gain by cellular adaptation. This explains that, after adaptation, the response to a uniform screen is very low. By opposition, in the ' 0 deg' experiment the situation is inverse (weak response during the preceding period, implying an augmentation of the cell's gain and a stronger response to a uniform screen). 


\subsubsection{Simulation with our model}

The results of our model are reproduced in Figure 10, for X cells and $\mathrm{Y}$ cells. $\mathrm{X}$ cell parameters were fixed on the same plausible values as for the Shapley and Victor experiment (Section 3.2). Note that parameters $\sigma_{C}$ and $\sigma_{S}$ (see caption to Figure 8), both in the Shapley-Victor experiment and in this one, were chosen to fit the receptive fields measurements for the original $\mathrm{X}$ cell in Figure 9, by the authors.

The model reproduces the absence of a 'null position' for Y cells, due to the post-synaptic pooling modeled in equation (17). We found that reproduction of $Y$ cell curves by a model requires the three following elements, in the right order: Additional transient in the IPL (12), Synaptic rectification (13) and Magnocellular pooling (17). See discussion in Section 4.2.2 for more details on the ordering of these three elements.

The model also reproduces the 'null position' typical of X cells. The slow decay of cell responses to static stimuli are also reproduced, with the correct time scale. This is due to the added effects of the IPL transient filter (14), also present to some extent in X cells, and mostly to the adaptation filter in the OPL (6) that provides the slow decay of the response over several hundreds of milliseconds. Note however that in our model, in any of the four experimental conditions, the cell sets back to the same firing rate at the end of the 'no grating' period: its ground firing rate. Parameters $T_{0}$ in (15) and $g^{L}$ in (16) were fixed to obtain a ground firing rate of around $50 \mathrm{~Hz}$.

As explained above (Section 3.3.1), this is not the case in real cells, that display the mark of a slow, non-linear adaptation to the grating that constituted the first part of the stimulus. Our simple adaptation scheme (6) fails to totally reproduce this complex phenomenon. First, because it is linear and that cellular adaptation is not. Second, because (6) is an adaptation to luminosity, whereas that adaptation observed here seems rather linked to the cell's own response to the grating that preceded (luminosity effects are slight, since the mean luminosity remains constant throughout the whole experiment).

One way of including in our model the adaptation effect observed here, could be to add a slow spike-frequency adaptation mechanism at the level of the spiking ganglion cells, e.g. as in Liu-Wang [20]. Such a non-linear scheme might complete the effects of our photoreceptor adaptation scheme (6).

\subsection{Results on real images}

To conclude our presentation of Virtual Retina, we present tests of the model on real images. In Section 3.4.1, we focus on the perceptual consequences of the non-linear gain control stage. In Section 3.4.2, we present a large-scale simulation of a retina, tested on a moving sequence, from input light to the output spikes emitted by the different channels of ganglion cells.

\subsubsection{Perceptual effect of contrast gain control}

Tests of the model on a whole image reveal the perceptual interest of the contrast gain control mechanism introduced in Section 2.3.1. It allows the retina to enhance edges, in a way very similar to traditional image processing techniques. 
This is illustrated in Figure 11. Our test image is shown in (a). It has been artificially modified to display a Michelson contrast of 1 on the left-hand side, and of 0.3 on the right-hand side. Image 11 (b) represents the equilibrium state of $I_{\text {Gang }}$ in a model without amacrine feedback, when $\lambda_{\mathrm{BA}}$ in (11) has been set to zero. By equilibrium state, we mean $I_{\text {Gang }}(x, y, t=\infty)$, a value being reached a few hundreds of milliseconds after image onset (like the equilibrium reached by the cell in Figure 10). In comparison, image 11 (c) represents $I_{\mathrm{Gang}}(x, y, \infty)$ in the retina model incorporating amacrine feedback. All three displays are normalized, with highest value in the image corresponding to color white.

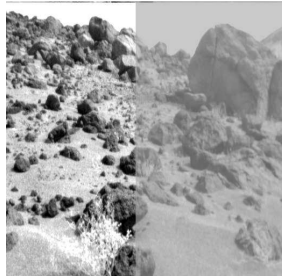

(a) Input

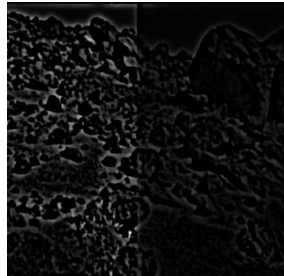

(b) No feedback

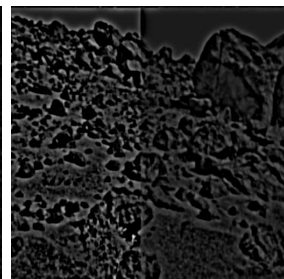

(c) With feedback

Figure 11: Effect of the contrast gain control on a real image. The contrast of the right-hand side part of the input image (a) was artificially reduced. Image (b) (resp (c)) represents the retinal signal $I_{\text {Gang }}$ (right before spike generation) without (resp. with) contrast gain control. In (c) the right-hand side part shows more details.

The divisive effect of amacrine cells is twofold. First, it is observed between the two zones of the image (left- and right-hand side), whose amplitudes become much more comparable thanks to contrast gain control. Second, it increases sensibility of the retina to 'uniform' zones of the image (such as the bottom right of the image). This is because the signal's amplification gain, at each point, is driven by the average level of contrast in the direct neighborhood. So perceptually, the gain control mechanism is close to some local enhancement techniques from image processing, such as local histogram equalization (see [13], chapter 3).

\subsubsection{Large-scale simulation of the model}

We show in Figure 12 the response of our model to an input video stimulation. Each column represents a different moment in time of the stimulation, while each line represents a different layer of the model. To display all possibilities of Virtual Retina, we chose a hybrid retina, with cell properties being those of a cat retina (with $\mathrm{X}$ and $\mathrm{Y}$ cells), but that displays a radial structure as in a primate retina, with spatial precision maximum in the fovea, and decreasing towards the periphery. The simulated retina had a diameter of $50 \mathrm{deg}$, corresponding to 250 pixels. The input sequence lasted $1.2 \mathrm{~s}$ of 'real time', corresponding to 56 frames. There were three ganglion layers of 30,000 spiking cells each. Total processing time was around $130 \mathrm{~s}$ ( 2 seconds per input frame). Model parameters are in the caption to Figure 12. 
The last three lines are reconstructions of retinal output signal from the set of spike trains emitted by the retina. They concern respectively X ON and OFF cells, and Y OFF cells. The reconstruction process consisted in a convolution of the spike trains with a temporal exponential kernel of time constant $20 \mathrm{~ms}$, associated to a circular spot whose diameter and intensity depended on the local density of cells at each location. Thus, a reconstructed sequence displays in each pixel a quantity close to the 'instantaneous firing rate for a cell located at this pixel'.

The foveated structure, ruled by sampling scheme (18), can be observed on all retinal images (except for the input light): There is better precision at the center of the retina. Signals $I_{C}, I_{S}$ and $I_{\mathrm{OPL}}$ illustrate the properties of the OPL filter $K_{\mathrm{OPL}}$ in (8): It is the difference of two low-passed versions of the sequence, and as such it takes strong values on image edges and on moving zones. Its biggest response is thus located on the edges of the walking characters. The perceptual effect of the contrast gain control scheme (9)-(11) can be observed by comparing signal $I_{\mathrm{OPL}}$, which is the result of a linear filter, and signal $V_{\text {Bip }}$ that has been non-linearly enhanced by a feedback with $g_{\mathrm{Am}}^{L}$. Signal $V_{\text {Bip }}$ presents much more contrast details. Finally, the positive and negative parts of signal $V_{\mathrm{Bip}}$ are coded respectively by $\mathrm{ON}$ and $\mathrm{OFF}$ ganglion cells. Y cells display a signal with less spatial precision than X cells, because of the supplementary synaptic pooling (17). Second, Y cells are only sensitive to temporal changes, so they only detect the moving characters. This is obtained by making the cells totally phasic with $w^{t r s}=1$ in (14), and by lowering the spontaneous firing rate of $\mathrm{Y}$ cells, through parameter $T_{0}$ in (15).

\section{Discussion}

\subsection{Non-linear gain controls}

A key feature of the present model is its detailed contrast gain control stage (9)-(11), and how it relates to experimental observations (Section 3.2). Here we discuss the biological relevance of our model: based on synaptic conductances (Section 4.1.1), and involving a feedback loop (Section 4.1.2). Then we compare it to other gain-control models that have been proposed at the level of the retina (Section 4.1.3).

\subsubsection{Synaptic conductances}

One strength of this model is its being based on a simple and yet biologically-plausible architecture mimicking synaptic conductances in the membrane of bipolar cells. Precise modeling of synapses with conductances is known to encompass divisive effects that are neglected by simpler models based on synaptic currents [7]. If a synaptic conductance $g(t)$ with Nernst potential $E$ is opened in a cellular membrane with potential $V(t)$, it creates a synaptic current

$$
g(t)(E-V(t))
$$

through the membrane, that contributes to cellular integration $d V / d t$. Such a current can be decomposed in a 'linear contribution' $g(t) E$ and a 'shunt contribution' $-g(t) V(t)$. If only the first term 


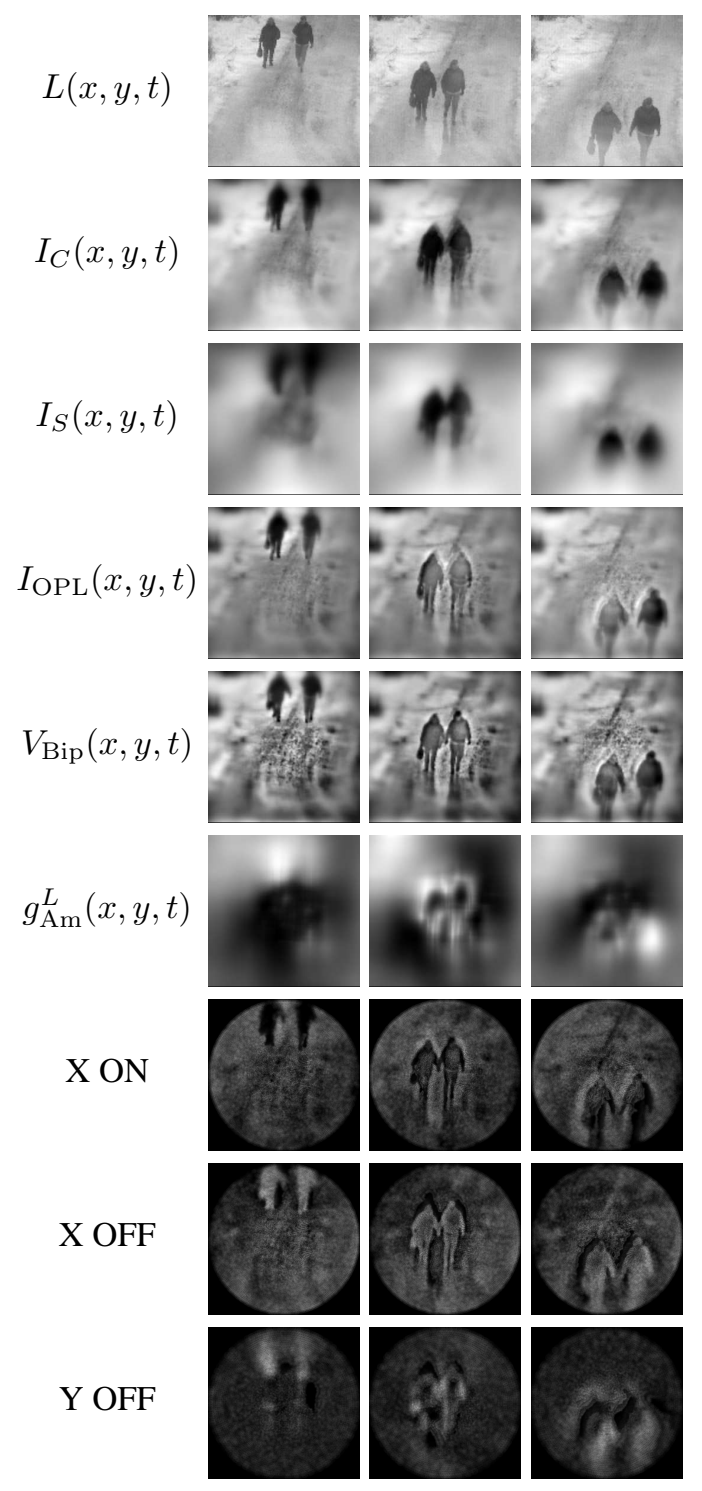

Figure 12: Large-scale simulation with cat $X$ and Y cells. Image size: 50 deg (250 pixels). Same parameters as in Figure 10, except $\sigma_{C}=0.3 \mathrm{deg}, \sigma_{S}=1 \mathrm{deg}, \sigma_{\mathrm{Am}}=2 \mathrm{deg}$, and for $\mathrm{Y}$ cells $\sigma_{\text {Gang }}=1 \mathrm{deg}, T_{0}=60 \mathrm{~Hz}$. Log-polar parameters: $R_{0}=10 \mathrm{deg}(50 \mathrm{pix}), K=0.2 \mathrm{deg}^{-1} .90,000$ spiking cells, simulation speed of around 1/100 real time (see text). 
is taken into account, then $V(t)$ can be linearly calculated from $g(t)$. The second term, by opposition, modifies non-linearly the instantaneous gain of membranar integration. We call it a 'shunt', or 'leak', term, because it does not depend on the Nernst potential $E$ associated to the ionic channel considered, so it has exactly the same divisive effect as an inert leak conductance in the membrane (associated to the cell's natural rest potential, taken as 0).

At the contrast gain control stage (9)-(11), our model focuses only on the shunting impact of amacrine-driven conductances onto bipolar cells, by implicitly considering that they have a null Nernst potential. On the contrary, at all other stages of retinal processing, the model uses linear filters, so it implicitly considers only the 'linear' contribution of synaptic transmissions. Amacrinedriven synapses are the only location of the model where both linear and shunting impacts are taken into account. However, both impacts are modeled at different stages: The shunting contribution of amacrine cells is modeled in (9)-(11), whereas their linear contribution is modeled in (12). This separation has two advantages. First, it allows to separate two conceptually different effects of amacrine cells. Second and more importantly, it is a more convenient way to functionally encompass the diversity of amacrine cells, their different types of synaptic conductances, and the diverse connectivity patterns implying amacrine cells [18]: Feedback to bipolar cells, gap junctions or synaptic inhibitions between amacrine cells (including between different subtypes), direct inhibition or feedback onto ganglion cells, etc.

\subsubsection{Feedback mechanism?}

Our gain control stage is based on a dynamical, non-linear feedback mechanism. Retinal processing does involve feedbacks and gain controls, because it must avoid saturation in response to a very wide range of input contrasts and luminosities. The phototransduction cascade in light receptors is the first and most striking example of a dynamical gain control. The chemical process is known to involve a complicated cascade of reactions where feedbacks play a large role [26].

The physiological importance of feedback mechanisms as compared to feed-forward inhibitions is that a feedback allows all physical magnitudes, such as cell potentials or synaptic currents, to always remain in a range that is allowed biologically. Briefly, if two magnitudes $A$ and $\bar{A}$ (average of the preceding) are calculated independently in the retina, and an output $A / \bar{A}$ is then somehow computed by the system, the problem remains of how big $A$ and $\bar{A}$ could possibly get before having the system to saturate. On the contrary, dynamical feedbacks from the ' $\bar{A}$ calculating' cells to the ' $A$ calculating' cells solve this saturation problem, because in that case the gain control directly operates at the level of the ' $A$ calculating' cells. Thus, contrast gain control in real retinas very likely implies a feedback mechanism.

There is evidence that a divisive invariance also exists at the level of the OPL $[31,1]$, probably between horizontal cells and light receptors. We suggest that horizontal cells provide shunting feedbacks to receptor cells in a way similar to that proposed here at the level of amacrine cells. This would provide a divisive invariance to the local luminosity at the level of receptors, in addition to the subtractive effect of horizontal cells already modeled in this article (equation 2).

The mathematical counterpart of a feedback-based model is the coupled dynamics that make the system harder to study theoretically. The temporal properties of our feedback loop (9)-(11) are still 
to be studied from a mathematical dynamical systems approach. This study is the next step of our research, to mathematically define the stability criteria of the system.

\subsubsection{Other models of gain control}

How does our model compare with other gain control models in the retina? The easiest way to implement a sub-linearity of retinal responses with respect to contrast, is that of standard models [4], also known as LNP (Linear, Nonlinear, Poisson) models. These models allow to choose, or experimentally determine (Chichilnisky [5]) a static non-linear function through which the filtered signal is passed before spike generation. Contrast gain control will then be represented by a compression part in the non-linear function, making the output under-linear with the input contrast. Advantage of this approach is its tractability from experimental data [5]. Inconveniences are its being non-dynamical (it cannot explain the temporal changes in filtering with contrast), its lack of spatial extent (the nonlinearity applies only cell by cell, so perceptual enhancements such as that presented in Section 3.4.1 are greatly reduced) and the fact that it does not explain at all where the non-linearity occurs in the retina.

Other non-linear models go further. Victor [32] proposes an empirical model that reproduces contrast gain control effects, thanks to a high-pass filtering stage whose time constant is a function of the recent values of contrast. The advantage is the relative simplicity of the resulting stage, that encompasses both our shunting effect (9)-(11) and our additional high-pass in the IPL (12). Counterpart is, again, the lack of biological location. Furthermore, the Victor model does not consider the spatial extent, and thus perceptual consequences, of the gain control. It is a direct influence to more recent models, such as the Y cell model of Enroth-Cugell and Freeman [9], or the Van Hateren model for primate Parasol cells [31].

On the contrary, the Bonin LGN model [1] proposes the idea of a divisive spatial surround. But it implements an invariance to local luminosity, so it accounts for different effects than our model, that handles contrast gain controls. Moreover, the modeled non-linearity has no effect on the temporal dynamical changes in the filtering kernel. For these two reasons, it cannot account for the contrast-based effects of Figure 7. Extending our shunting feedback model to the OPL, between light receptors and horizontal cells (see Section 4.1.2), probably provides a divisive invariance to luminosity close to that of the Bonin model.

To sum up, our model makes the synthesis of two 'trends' of gain control models: those based on the temporal expression of the gain control and those based on its spatial expression. Furthermore, our model goes further in the biological location of its components, and uses the concept of feedback, as we claim it does happen in real retinas.

\subsection{Subclasses of ganglion cells}

Let us now discuss classification issues about ganglion cells, and how our model reproduces this classification. Section 4.2.1 is a short reminder of the main types of ganglion cells in cat and in primate retinas. Section 4.2.2 is a further discussion on how to build a model for cat $\mathrm{Y}$ cells. 


\subsubsection{Ganglion cells in cat and primate retinas}

Names and classification of ganglion cells vary according to the species considered, and to the classification medium (morphology or physiology) [18, 23]. The goal of this Section is not to review all types, but just to give landmarks about retinal physiology and how our simulator relates to them.

In the cat retina, two physiological types of ganglion cells are very well known: $\mathrm{X}$ cells and $\mathrm{Y}$ cells, as described in Section 3.3.1. In a morphological classification, they are termed respectively $\beta$ and $\alpha$ type cells. Both types of cells display a strong contrast gain control [28], although the effect is stronger in $\mathrm{Y}$ cells. Y cells are more phasic, $\mathrm{X}$ cells more tonic. Finally, the response of $\mathrm{Y}$ cells cannot be modeled by linear spatial summation (3.3.1). Our model can account for both $\mathrm{X}$ and $\mathrm{Y}$ types of cellular response. Many other types of ganglion cells have been found in the cat retina (such as $W$ cells), with functionalities more or less known [23]. Some are sensitive to illumination, others are tuned to directional movement, etc. Our model does not intend to reproduce these other subtypes, although we believe that for some of them, modeling is relatively straightforward from what is presented in this article.

In the primate retina also, two physiological types of ganglion cells have received much more attention than the others, they are Midget and Parasol cells. Midget cells are very precise spatially (small receptive field) and code for color oppositions. They are known to display little contrast gain control [16]. They are connected to the Parvocellular pathway of the LGN, which is supposed to be in charge of precise shape detection. Parasol cells have a wider receptive field, are not sensitive to color but very sensitive to contrast, and display a strong contrast gain control effect. They are connected to the Magnocellular pathway of the LGN, which is supposed to be in charge of movement detection and broad scene analysis. Elements suggest that Midget cells constitute a new channel of visual information possessed only by primates, whereas Parasol cells are a common feature shared with other mammals, being close for example to cat $\mathrm{X}$ and $\mathrm{Y}$ cells $[16,23]$. Following this hypothesis, Virtual Retina can reproduce primate Parasol cells with efficiency. Midget cells can also be reproduced in their achromatic features; but color oppositions are not handled yet by our model. Finally, as explained already, Virtual Retina also handles the foveated structure typical to primate retinas.

Table 1 summarizes plausible orders of magnitude for the model parameters that must vary according to the type of cell modeled. Values for $\sigma_{C}$ and $\sigma_{S}$ are taken from the literature. For other parameters of cat cells, we give plausible orders of magnitude, that provided good fit to data. Other parameters of primate cells are only plausible suggestions that have not been tested, but respect the scaling from cat to primate retina, and the characteristics of primate cells explained in the above paragraph: Parasol cells can behave like cat X or Y cells, and Midget cells display no contrast gain control.

\subsubsection{Y cell model}

Cat Y cells are a long-known type of ganglion cells that have been intensively studied physiologically. First, their high sensitivity to contrast and their fast axon make them a plausible input to movement detection tasks in the cortex. Second, their responses display a spatial non-linearity that 


\begin{tabular}{lcccc}
\hline \hline Parameter & Cat X & Cat $\mathrm{Y}$ & Parasol & Midget \\
\hline$\sigma_{C}(\mathrm{deg})$ & 0.5 & 0.5 & $0.05($ fov) & 0.05 (fov) \\
$\sigma_{S}(\mathrm{deg})$ & 1.5 & 1.5 & $0.15($ fov) & 0.15 (fov) \\
$\sigma_{A}(\mathrm{deg})$ & 1.5 & 1.5 & $0.15($ fov) & 0.15 (fov) \\
$\sigma_{G}(\mathrm{deg})$ & 0 & 1.5 & $0-0.15($ fov) & 0 (fov) \\
$\lambda_{\mathrm{BA}}(\mathrm{Hz})$ & 100 & 100 & 100 & 0 \\
$g_{\mathrm{BA}}(\mathrm{Hz})$ & 5 & 5 & 5 & 50 \\
$w^{t r s}$ & 0.7 & 1 & $0.7-1$ & 0.7 \\
$\lambda_{\mathrm{BG}}(\mathrm{Hz})$ & 100 & 400 & $100-400$ & 100 \\
\hline \hline
\end{tabular}

Table 1: Model parameters that vary according to species and pathway. Plausible values for other parameters are in caption to Figure 8.

make them harder to model than X cells. Third, their wide dendritic trees and cell bodies were easy targets for experimentalists' electrodes [23].

The idea that the spatial non-linearity of $Y$ cells might be due to a pooling from some presynaptic cells dates back to the discovery of 'linear subunits' in the receptive fields of Y cells by Hochstein and Shapley [15]. Authors such as Enroth-Cugell and Freeman [9] have exploited the idea to build a model for Y cells, based on the combination of a static non-linearity, a high-pass linear filter and a spatial pooling. However, if their model performs fine on drifting gratings, it fails to reproduce correctly a disappearing grating experiment as in Figure 9, because of a wrong order of the three elements constitutive of the model. Let us now discuss this order, with the illustration presented in Figure 13.

First, the spatial pooling must be applied after the static non-linearity (termed 'rectification' in Figure 13). Only this order gives rise to the absence of a 'null position' for the $\mathrm{Y}$ cell (3.3.1), and is at the core of the 'linear subunit' model. Second, in order to reproduce the $\mathrm{Y}$ cell experiment in Figure 9, the high-pass filter must be applied before the static non-linearity, which is not the case in the Enroth-Cugell and Freeman model. On top of Figure 13 are schematic representations of two bipolar signals that are going to influence the considered Y cell: Signal from bipolar cells at the same retinal location as the $\mathrm{Y}$ cell (purple, short dashes), and signal from bipolar cells at neighboring locations (green, long dashes), where the spatial grating is counter-phased, but that will also influence the considered Y cell because of the spatial pooling. From this situation, our model can correctly generate positive activity bursts of the $\mathrm{Y}$ cell signal (orange, solid line) both at onset and offset of the grating. On the contrary, because of a wrong order of elements, the Enroth-Cugell and Freeman model necessarily implies opposite reactions of the $\mathrm{Y}$ cell at onset and offset of the grating (see Figure 13), as opposed to experimental results of Figure 9. 


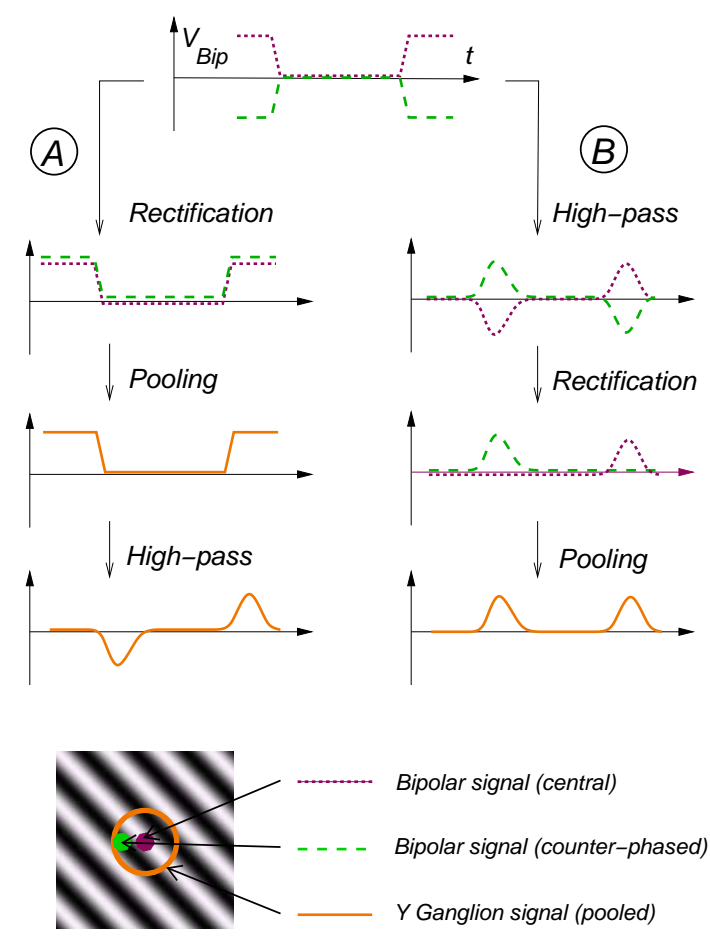

Figure 13: Two models used to generate the spatial non-linearity of cat $\mathrm{Y}$ cells, schematically tested on the appearing grating stimulus of Figure 9 (see text). Only our model can reproduce the two bursts of activity in the output. A: Y cell model of Enroth-Cugell and Freeman [9]. B: Our model.

\section{Conclusion}

In this article we proposed a model for achromatic retinal processing in mammalian retinas, from input light to emitted spikes. The model is also implemented in a software allowing large-scale simulations of spiking cells, that is available as an open-source software. The model takes into account two important non-linear effects of the retina: dynamical contrast gain control, and spatial non-linearity of cat Y cells.

The three stages of the model have been associated to morphological layers of the retina, in an effort to understand how the characteristics of retinal output arise along the pathway. The first stage of the model corresponds to the Outer Plexiform Layer, the first layer of synaptic interactions in the retina. We modeled it as a spatio-temporal linear filter, whose being not separable in time and space provides a better detection of image edges and of movements. The second stage of the model corresponds to the Inner Plexiform Layer, the second layer of synaptic interactions in the retina. We located at this level the appearance of contrast gain control in the retina, through a dynamical 
shunting feedback from amacrine cells, which constitutes the most original contribution of the paper in terms of retinal modeling. The third stage of the model corresponds to the ganglion cell layer, which contains the output spiking cells of the retina. We modeled it as an array of simple spiking LIF neurons, with biological sources of noise and the possibility to account for the spatial non-linearity of cat Y cells.

Our associated simulation software, Virtual Retina, can emulate up to around 100,000 cells with a processing speed of about 1/100 real time, which makes it appropriate for large-scale simulations of the visual cortex (and possibly LGN) that require a realistic input from the retina. It is still an evolving software, that might include future evolutions such as: realistic eye movements (pure rotation), chromatic oppositions, and fitting of parameters to build a feed-forward model of LGN.

\section{Acknowledgments}

This work was partially supported by the EC IP project FP6-015879, FACETS.

\section{Appendix}

Let us comment further the choice of the convolution by kernel $K_{\sigma, \tau}(x, y, t)$ to model signal averaging in a layer of cells (see Section 2.1.2). Suppose that a layer of retinal cells, described by the spatially continuous potential $V(x, y, t)$, is linearly driven by an input synaptic current $I(x, y, t)$. Then, $V$ can always be linearly calculated from $I$, through an impulse response $K(x, y, t)$ :

$$
V(x, y, t)=K * I(x, y, t),
$$

where symbol $*$ represents spatio-temporal convolution. Because neurons are small 'RC' circuits, $K$ is temporally low-pass, with a term in $\exp (-t / \tau)$. However, the precise expression of $K$ depends on the type of spatial averaging being modeled. There are two effects:

- Averaging because of the cells' dendritic spread is well modeled by a static spatial Gaussian kernel, leading to filter $K_{\sigma, \tau}(x, y, t)$ in (1).

- Averaging by gap junctions between neighboring cells can be expressed either in a discretecell approach [21, 14] or in a continuous setting with Laplacian-like operators [24, 19]. Both approaches lead approximately to the same impulse response

$$
K_{\text {gap junctions }}(x, y, t)=G \sqrt{2 \mathcal{G} t}(x, y) \exp (-t / \tau),
$$

where $\mathcal{G}$ is a constant measuring the two-dimensional density of gap junctions.

One can verify that both filters (1) and (21) react as spatio-temporal low-pass filters, with very similar characteristics in the Fourier domain. Filter (21) is a bit harder to handle mathematically, because of the influence of time $t$ on the spatial Gaussian kernel. For this reason, we model all low-pass effects, including effect of gap junctions, with the single filter (1). 


\section{References}

[1] V. Bonin, V. Mante, and M. Carandini. The suppressive field of neurons in Lateral Geniculate Nucleus. Journal of Neuroscience, 25(47):10844-10856, November 2005.

[2] D. Cai, G. C. Deangelis, and D. Freeman. Spatiotemporal receptive field organization in the lateral geniculate nucleus of cats and kittens. Journal of Neurophysiology, 78(2):1045-1061, August 1997.

[3] M. Carandini. Visual cortex: fatigue and adaptation. Current Biology, 10, 2000.

[4] M. Carandini, J. B. Demb, V. Mante, D. J. Tollhurst, Y. Dan, B. A. Olshausen, J. L. Gallant, and N. C. Rust. Do we know what the early visual system does? Journal of Neuroscience, 25(46):10577-10597, November 2005.

[5] E. J. Chichilnisky. A simple white noise analysis of neuronal light responses. Network: Comput. Neural Syst., 12:199-213, 2001.

[6] D. Dacey and M. Petersen. Dendritic field size and morphology of midget and parasol ganglion cells of the human retina. Proc Natl Acad Sci, 89:9666-70, 1992.

[7] P. Dayan and L. F. Abbott. Theoretical Neuroscience : Computational and Mathematical Modeling of Neural Systems. MIT Press, 2001.

[8] J. B. Demb, K. Zaghloul, L. Haarsma, and P. Sterling. Bipolar cells contribute to nonlinear spatial summation in the brisk-transient (Y) ganglion cell in mammalian retina. Journal of Neuroscience, 21(19):7447-7454, October 2001.

[9] C. Enroth-Cugell and A. W. Freeman. The receptive-field spatial structure of cat retinal Y cells. Journal of Physiology, 384(1):49-79, 1987.

[10] C. Enroth-Cugell and J. G. Robson. The contrast sensitivity of retinal ganglion cells of the cat. J Physiol, 187:517-552, 1966.

[11] H. Erwin. Web : http://scat-he-g4.sunderland.ac.uk/ harryerw/phpwiki/index.php/tonicphasic, 2004.

[12] N. Gazeres, L. Borg-Graham, and Y. Fregnac. A model of non-lagged X responses to flashed stimuli in the cat lateral geniculate nucleus. Visual Neuroscience, 1998.

[13] Rafael C. Gonzalez and Richard E. Woods. Digital Image Processing. Addison Wesley, 3 edition, 1992.

[14] J. Herault. A model of colour processing in the retina of vertebrates: from photoreceptors to colour opposition and colour constancy phenomena. Neurocomputing, 12:113-129, 1996.

[15] S. Hochstein and R. M. Shapley. Linear and nonlinear spatial subunits in Y cat retinal ganglion cells. J Physiol, 262:265-284, 1976. 
[16] Ehud Kaplan and Ethan Bernadete. The dynamics of primate retinal ganglion cells. Progress in Brain Research, 134:1-18, 2001.

[17] J. Keat, P. Reinagel, R. C. Reid, and M. Meister. Predicting every spike: a model for the responses of visual neurons. Neuron, 30:803-817, 2001.

[18] H. Kolb, E. Fernandez, and R. Nelson. Webvision : the Organization of the Retina and Visual System. Web : http://webvision.med.utah.edu/, 2001.

[19] T. D. Lamb. Spatial properties of horizontal cell responses in the turtle retina. J Physiol, 263(2):239-55, 1976.

[20] Y.H. Liu and X.J. Wang. Spike-frequency adaptation of a generalized leaky integrate-and-fire model neuron. Journal of Computational Neuroscience, 10(1):25-45, January 2001.

[21] M. A. Mahowald and C. Mead. The silicon retina. Sci. Am., 264(5):76-82, 1991.

[22] Susana Martinez-Conde, Stephen L. Macknik, and David H. Hubel. The role of fixational eye movements in visual perception. Nature Reviews Neuroscience, 5:229-240, 2004.

[23] R. Masland. The fundamental plan of the retina. Nature neuroscience, 4(9), September 2001.

[24] K. I. Naka and W. A. Rushton. The generation and spread of s-potentials in fish (cyprinidae). J Physiol, 192(2):437-61, September 1967.

[25] S. Nirenberg and M. Meister. The light response of retinal ganglion cells is truncated by a displaced amacrine circuit. Neuron, 18:637-650, 1997.

[26] A. Polans, W. Baehr, and K. Palczewski. Turned on by $\mathrm{Ca}^{2+}$ ! The physiology and pathology of $\mathrm{Ca}^{2+}$-binding proteins in the retina. Trends in Neurosciences, 19(12):547-554, December 1996.

[27] J. L. Schnapf, B. J. Nunn, M. Meister, and D. A. Baylor. Visual transduction in cones of the monkey macaca fascicularis. J Physiol, 427(1):681-713, 1990.

[28] R. M. Shapley and J. D. Victor. The effect of contrast on the transfer properties of cat retinal ganglion cells. The Journal of Physiology, 285(1):275-298, 1978.

[29] S. Tan, J. Dale, and A. Johnston. Performance of three recursive algorithms for fast spacevariant gaussian filtering. Real-Time Imaging, 2003.

[30] J.M. Valeton and D. Van Norren. Light adaptation of primate cones : An analysis based on extracellular data. Vision Research, 23(12):1539-1547, 1983.

[31] J. H. van Hateren, L. Rüttiger, H. Sun, and B. B. Lee. Processing of natural temporal stimuli by macaque retinal ganglion cells. J. Neuroscience, 22(22):9945-9960, 2002.

[32] J. D. Victor. The dynamics of the cat retinal X cell centre. The Journal of Physiology, 386(1):219-246, 1987. 


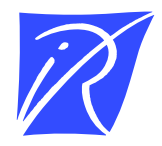

\section{Unité de recherche INRIA Sophia Antipolis 2004, route des Lucioles - BP 93 - 06902 Sophia Antipolis Cedex (France)}

Unité de recherche INRIA Futurs : Parc Club Orsay Université - ZAC des Vignes 4, rue Jacques Monod - 91893 ORSAY Cedex (France)

Unité de recherche INRIA Lorraine : LORIA, Technopôle de Nancy-Brabois - Campus scientifi que 615, rue du Jardin Botanique - BP 101 - 54602 Villers-lès-Nancy Cedex (France)

Unité de recherche INRIA Rennes : IRISA, Campus universitaire de Beaulieu - 35042 Rennes Cedex (France)

Unité de recherche INRIA Rhône-Alpes : 655, avenue de l'Europe - 38334 Montbonnot Saint-Ismier (France)

Unité de recherche INRIA Rocquencourt : Domaine de Voluceau - Rocquencourt - BP 105 - 78153 Le Chesnay Cedex (France) 